\title{
Association between Cross-sectional Areas of Lumbar Muscles on Magnetic Resonance Imaging and Chronicity of Low Back Pain
}

\author{
Hak Il Lee, M.D., Junyoung Song, M.D., Hee Song Lee, M.D., Jin Young Kang, M.D. ', \\ Minyoung Kim, M.D., Ph.D., Ju Seok Ryu, M.D., Ph.D.
}

Department of Rehabilitation Medicine, CHA Bundang Medical Center, CHA University, Seongnam 463-712,

${ }^{1}$ Sanbon Medical Center, Wonkwang University, Gunpo 435-040, Korea

Objective To investigate the prognostic value of cross-sectional areas (CSA) of paraspinal (multifidus and erector spinae) and psoas muscles on magnetic resonance imaging (MRI) in chronicity of low back pain.

Method Thirty-eight subjects who visited our hospital for acute low back pain were enrolled. Review of their medical records and telephone interviews were done. Subjects were divided into two groups; chronic back pain group (CBP) and a group showing improvement within 6 months after onset of pain (IBP). The CSA of paraspinal and psoas muscles were obtained at the level of the lower margin of L3 and L5 vertebrae using MRI.

Results CSA of erector spinae muscle and the proportion of the area to lumbar muscles (paraspinal and psoas muscles) at L5 level in the CBP group were significantly smaller than that of the IBP group $(\mathrm{p}<0.05)$. The mean value of CSA of multifidus muscle at L5 level in the CBP group was smaller than that of the IBP group, but was not statistically significant ( $p>0.05$ ). CSA of psoas muscle at L5 level and all values measured at L3 level were not significantly different between the groups ( $\mathrm{p}>0.05)$.

Conclusion CSA of erector spinae muscle at the lower lumbar level and the proportion of the area to the lumbar muscles at the L5 level can be considered to be prognostic factors of chronicity of low back pain.

Key Words Low back pain, Magnetic resonance imaging, Cross-sectional area, Muscles

Received June 9, 2011; Accepted September 15, 2011

Corresponding author: Ju Seok Ryu

Department of Rehabilitation Medicine, CHA Bundang Medical Center, CHA University, 351 Yatap-dong, Bundang-gu, Seongnam 463-712, Korea Tel: +82-31-780-6281, Fax: +82-31-780-6206, E-mail: Jseok337@cha.ac.kr (c) This is an open-access article distributed under the terms of the Creative Commons Attribution Non-Commercial License (http:// creativecommons.org/licenses/by-nc/3.0) which permits unrestricted noncommercial use, distribution, and reproduction in any medium, provided the original work is properly cited.

Copyright $\odot 2011$ by Korean Academy of Rehabilitation Medicine

\section{INTRODUCTION}

Low back pain is one of the most common diseases in society, with about $80 \%$ of the adult population experiencing it at least once in their life-time. ${ }^{1}$ It is also the second largest reason for the outpatient visits. ${ }^{2}$ Acute low back pain usually resolves within 6 to 12 weeks. But, many of those who recover experience recurrence of the symptoms, which can become chronic. ${ }^{1,3}$ Most of the patients with low back pain are symptomatic 1-year later, and only $25 \%$ of patients fully recover without any low 
back pain disability. ${ }^{4}$ Low back pain, therefore, places a considerable restriction on functional and occupational activities of patients, which affects society and the economy, making a relevant treatment for the disease even more important. ${ }^{1,3,4}$

Exercise for low back pain has focused on lumbar stabilization and core strengthening. ${ }^{5-7}$ Especially, the multifidus muscle, one of the paraspinal muscles, is attached to the spine at every segment to provide stability to the spine. As such, it is sensitive to pathologic change. Lumbar stabilization exercises that strengthen the multifidus muscles reduce low back pain and its recurrence rate. ${ }^{5-7}$

Studies have focused on identifying lumbar muscle changes as an index for functional disability in low back pain patients. Multifidus muscle atrophy and reduction of their cross-sectional areas (CSA) has been descvribed..$^{8-11}$ Kader et al. ${ }^{8}$ analyzed the lumbar magnetic resonance imaging (MRI) data of 78 chronic low back pain patients, and reported that $80 \%$ of the patients showed multifidus muscle atrophy. Danneels et al. ${ }^{9}$ reported in their study using lumbar computed tomography (CT) that the CSA of the multifidus muscles of patients with chronic low back pain was smaller than that of the healthy control group, with similar results from the ultrasound imaging studies of Hides et al. ${ }^{10}$ and Wallwork et al. ${ }^{11}$ These studies mostly focused on the changes involving the multifidus muscles. Some reports addressed the changes in psoas muscle or paraspinal muscles. ${ }^{12,13}$ Parkkola et al. ${ }^{12}$ noted that in patients with chronic low back pain, the psoas muscles and the paraspinal muscles were smaller compared to those of the healthy control group. Dangaria and Naesh ${ }^{13}$ observed that patients with ipsilateral disc herniation, which is a cause of unilateral sciatica, showed reduced CSA of the ipsilateral psoas muscles.

Studies conducted to date have focused on the comparison of the CSA of the lumbar muscles between patients with chronic low back pain and the healthy asymptomatic subjects, while few studies have addressed the association between chronicity of the acute low back pain and the CSA of lumbar muscles. The aim of this study was to investigate the association between the chronicity of adult patients with no past history of low back pain from acute low back pain to chronic back pain, and the CSA of lumbar muscles upon MRI.

\section{MATERIALS AND METHODS}

\section{Subjects}

Between January 2006 and December 2009, 491 patients who visited our Department of Rehabilitation Medicine for acute low back pain and received lumbar MRI were enrolled. Inclusion criteria were: 1) age between 20 and 40 years; 2) no past history of low back pain; 3 ) MRI performed within 1 month from the first appearance of the symptom; 4) ability to ambulate and be treated as an outpatient; and 5) no abnormal findings (disc herniation, disc bulging, spinal stenosis, spondylolysis, spondylolisthesis, scoliosis, facet arthritis, spinal fracture and disc degeneration) on lumbar MRI and normal official radiology report. Forty nine patients satisfied the inclusion criteria. A telephone interview was initiated with each of these patients to discuss height, weight and duration of low back pain. Of the 49 subjects, 11 who refused a telephone interview or who changed their telephone numbers were excluded from the study. Finally, 38 subjects (22 males and 16 females; mean age $29.1 \pm 4.6$ years) participated in the study. The subjects were divided into two groups by their duration of low back pain: chronic back pain (CBP) group (13 subjects, 10 males and three females; mean age 29.6 \pm 4.1 years) and the improved back pain (IBP) group (25 subjects, 12 males and 13 females; mean age $28.8 \pm 4.9$ years). The CBP group was defined as patients who experienced low back pain for more than 6 months and the IBP group was defined as patients who recovered low back pain within 6 months.

\section{Methods}

Through the telephone interviews, the height, body weight, and the duration of the low back pain were investigated. The body weight $(\mathrm{kg})$ was divided by the square of the height $\left(\mathrm{m}^{2}\right)$ to calculate the body mass index (BMI).

The CSA of paraspinal (multifidus and erector spinae) and psoas muscles were obtained from axial T2 weighted images, which was superior in distinguishing muscle from fat, at the lower margin of L3 and L5 vertebrae using the Marosis m-view version 5.4 program (Fig. 1). The CSA of multifidus and erector spinae muscles in the paraspinal muscles were separately measured on the bilateral sides. The CSA of psoas muscles were also 

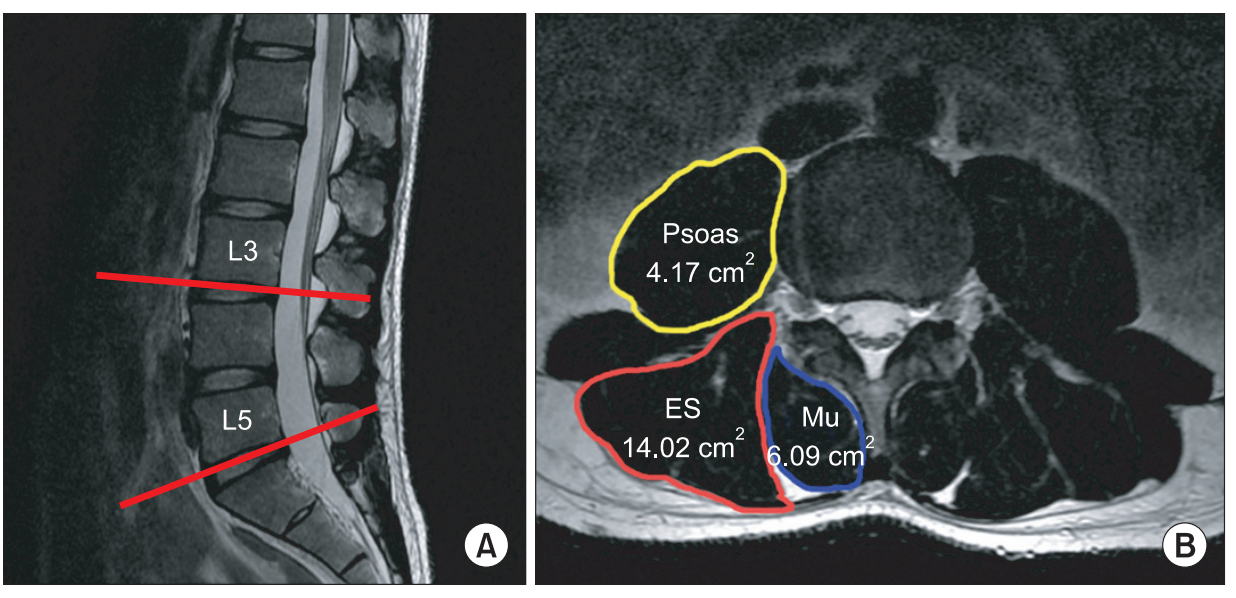

Fig. 1. The figure (A) shows sagittal view of lumbar spine. The CSA of paraspinal and psoas muscles were obtained on axial T2 weighted images at the lower margin of L3 (B) and L5 vertebrae using the Marosis $\mathrm{m}$-view version 5.4 program. Mu: Multifidus, ES: Erector spinae.

measured on the bilateral sides. The proportions of the multifidus, erector spinae and psoas muscles to the lumbar muscles (paraspinal and psoas muscles) were calculated by dividing each CSA of the muscles by the sum of the CSA of the paraspinal muscles and the psoas muscles.

Statistical analysis was performed using SPSS 19.0. Mann-Whitney test was used to compare the differences between the two groups, in terms of CSA and proportion of each muscle. Statistical significance was considered at $\mathrm{p}$-value $<0.05$. All data are presented as mean \pm standard deviation.

\section{RESULTS}

The age, height, weight and BMI were not significantly different between the two groups (Table 1).

The CSA of erector spinae muscle at the lower margin of the L5 vertebrae in the CBP group (Right: $7.8 \pm 2.4 \mathrm{~cm}^{2}$, Left: $\left.8.0 \pm 2.7 \mathrm{~cm}^{2}\right)$ was significantly smaller than that of IBP group (Right: $11.2 \pm 3.6 \mathrm{~cm}^{2}$, Left: $11.8 \pm 3.6 \mathrm{~cm}^{2}$ ) $(\mathrm{p}<0.05)$ (Table 2).

The proportion of erector spinae muscle to lumbar muscles at the lower margin of the L5 vertebrae in the CBP group (Right: $0.22 \pm 0.05 \mathrm{~cm}^{2}$, Left: $0.23 \pm 0.05 \mathrm{~cm}^{2}$ ) was significantly smaller than that of the IBP group (Right: $0.29 \pm 0.06 \mathrm{~cm}^{2}$, Left: $\left.0.31 \pm 0.06 \mathrm{~cm}^{2}\right)(\mathrm{p}<0.05)$. However, there was no statistical significance in the difference between the two groups at the lower margin of the L3 vertebrae (Table 3 ).

The mean CSA of the multifidus muscle measured at the lower margin of the L5 vertebrae was smaller in the CBP group (Right: $10.4 \pm 1.7 \mathrm{~cm}^{2}$, Left: $10.5 \pm 1.7 \mathrm{~cm}^{2}$ ) than that
Table 1. Demographic Data of the Subjects.

\begin{tabular}{lcrc}
\hline & IBP group & CBP group & p-value \\
\hline Age (years) & $28.88 \pm 4.90$ & $29.69 \pm 4.10$ & 0.58 \\
Height $(\mathrm{m})$ & $1.69 \pm 0.09$ & $1.71 \pm 0.06$ & 0.44 \\
Weight $(\mathrm{kg})$ & $63.84 \pm 13.20$ & $66.38 \pm 10.2$ & 0.54 \\
BMI $\left(\mathrm{kg} / \mathrm{m}^{2}\right)$ & $22.08 \pm 2.60$ & $22.46 \pm 2.10$ & 0.58 \\
\hline
\end{tabular}

CBP: Chronic back pain, IBP: Improved back pain, BMI: Body mass index

Table 2. CSA of Paraspinal and Psoas Muscles at the L5 Level

\begin{tabular}{lllll}
\hline & & IBP group & CBP group & p-value \\
\hline Multifidus $\left(\mathrm{cm}^{2}\right)$ & Right & $11.60 \pm 1.80$ & $10.40 \pm 1.70$ & 0.097 \\
& Left & $11.60 \pm 2.10$ & $10.50 \pm 1.70$ & 0.272 \\
$\begin{array}{l}\text { Erector spinae } \\
\left(\mathrm{cm}^{2}\right)\end{array}$ & Right & $11.20 \pm 3.60$ & $7.80 \pm 2.40$ & $0.004^{*}$ \\
& & & & \\
Psoas $\left(\mathrm{cm}^{2}\right)$ & Left & $11.80 \pm 3.60$ & $8.00 \pm 2.70$ & $0.002^{*}$ \\
& Right & $14.60 \pm 4.50$ & $15.80 \pm 4.00$ & 0.377 \\
& Left & $13.90 \pm 4.10$ & $15.40 \pm 3.20$ & 0.222 \\
\hline
\end{tabular}

CBP: Chronic back pain, IBP: Improved back pain, CSA: Cross-sectional areas

${ }^{*} \mathrm{p}<0.05$

of IBP group (Right: $11.6 \pm 1.8 \mathrm{~cm}^{2}$, Left: $11.6 \pm 2.1 \mathrm{~cm}^{2}$ ), but the difference was not statistically significant. Rather, the mean CSA of the psoas muscles was larger in the CBP group (Right: $15.8 \pm 4.0 \mathrm{~cm}^{2}$, Left: $15.4 \pm 3.2 \mathrm{~cm}^{2}$ ) than in the IBP group (Right: $14.6 \pm 4.5 \mathrm{~cm}^{2}$, Left: $13.9 \pm 4.1 \mathrm{~cm}^{2}$ ) with no statistically significant difference between the two groups (Table 2).

The CSA of erector spinae and psoas muscles measured at the lower margin of L3 vertebrae were rather larger in 
Table 3. Proportion of Each Muscle to Lumbar Muscles*

\begin{tabular}{lllccc}
\hline & & IBP group & CBP group & p-value \\
\hline At L3 level & Multifidus (\%) & Right & $0.21 \pm 0.05$ & $0.19 \pm 0.03$ & 0.246 \\
& & Left & $0.18 \pm 0.04$ & $0.17 \pm 0.03$ & 0.329 \\
& Erector spinae (\%) & Right & $0.49 \pm 0.04$ & $0.49 \pm 0.04$ & 1.000 \\
& & Left & $0.52 \pm 0.04$ & $0.51 \pm 0.05$ & 0.627 \\
\hline \multirow{2}{*}{ At L5 level } & Psoas (\%) & Right & $0.29 \pm 0.05$ & $0.31 \pm 0.04$ & 0.314 \\
& & Left & $0.29 \pm 0.04$ & $0.30 \pm 0.04$ & 0.411 \\
& Multifidus (\%) & Right & $0.31 \pm 0.04$ & $0.30 \pm 0.04$ & 0.671 \\
& & Left & $0.31 \pm 0.04$ & $0.31 \pm 0.04$ & 0.832 \\
& Erector spinae (\%) & Right & $0.29 \pm 0.06$ & $0.22 \pm 0.05$ & $0.006^{\dagger}$ \\
& & Left & $0.31 \pm 0.06$ & $0.23 \pm 0.05$ & $0.000^{\dagger}$ \\
& Psoas (\%) & Right & $0.38 \pm 0.06$ & $0.46 \pm 0.07$ & $0.006^{\dagger}$ \\
\hline
\end{tabular}

CBP: Chronic back pain, IBP: Improved back pain

${ }^{*}$ Lumbar muscles include multifidus, erector spinae and psoas, ${ }^{\dagger} \mathrm{p}<0.05$

Table 4. CSA of Paraspinal and Psoas Muscles at the L3 Level

\begin{tabular}{llccc}
\hline & & $\begin{array}{c}\text { IBP } \\
\text { group }\end{array}$ & $\begin{array}{c}\text { CBP } \\
\text { group }\end{array}$ & p-value \\
\hline Multifidus $\left(\mathrm{cm}^{2}\right)$ & Right & $6.70 \pm 1.50$ & $6.70 \pm 1.00$ & 0.976 \\
& Left & $6.00 \pm 1.40$ & $6.20 \pm 1.20$ & 0.584 \\
$\begin{array}{l}\text { Erector spinae } \\
\left(\mathrm{cm}^{2}\right)\end{array}$ & Right & $16.60 \pm 5.20$ & $17.60 \pm 3.80$ & 0.411 \\
& & & & \\
Psoas $\left(\mathrm{cm}^{2}\right)$ & Left & $17.60 \pm 5.20$ & $18.60 \pm 4.20$ & 0.411 \\
& Right & $9.90 \pm 3.80$ & $11.50 \pm 3.60$ & 0.314 \\
& Left & $10.00 \pm 3.80$ & $11.30 \pm 3.20$ & 0.259 \\
\hline
\end{tabular}

CBP: Chronic back pain, IBP: Improved back pain, CSA: Cross-sectional areas

the CBP group than in the IBP group. But, the difference between the two groups did not show any statistical significance. The CSA of the multifidus muscles at the lower margin of the L3 vertebrae also was not significantly different between the two groups (Table 4).

\section{DISCUSSION}

Atrophy of the paraspinal muscles can be evaluated using ultrasound, CT, and MRI. MRI is hindered by its expense. But, it offers higher image quality for soft tissues and is superior for examination in certain anatomical locations. MRI has thus been used in a number of studies, including this study. ${ }^{8,12,13}$ Until now, most studies have focused on the comparison of the CSA of lumbar muscles between the patients with chronic back pain and healthy asymptomatic subjects to investigate the correlation between muscular atrophy or asymmetry and severity of symptoms or lumbar lesions, for example in lumbar disc herniation. ${ }^{9-13}$ The present study excluded patients who showed any lumbar lesions on MRI, to eliminate lesion effects that could be related to chronicity of low back pain. Such exclusion was also made for the investigation of the association between the chronicity of the adult patients with no past history of low back pain from acute low back pain to a chronic one and the CSA of lumbar muscles on MRI.

Bergmark $^{14}$ classified the muscles that control the trunk into two groups. The first group is the muscles that are directly attached to the lumbar vertebrae and provide spine segmental stability, which includes lumbar multifidus, transversus abdominis, and internal oblique muscles. The second group is composed of the large torque-producing muscles with no segmental attachment to the lumbar vertebrae. These muscles control gross trunk movement and provide general trunk stability. This group includes erector spinae, rectus abdominis, and external oblique muscles. Among these muscles, the lumbar erector spinae muscle consists of the longissimus and iliocostalis muscles. The longissiums muscle originates from the lumbar transverse and accessory processes, and inserts onto the ventral surface of the posterior superior iliac spine. The iliocostalis 
muscle originates from the adjacent middle layer of the thoracolumbar fascia and the tips of the lumbar transverse processes and inserts onto the ventral edge of the iliac crest. ${ }^{15}$ With these anatomical characteristics, the erector spinae muscle has a longer lever arm than the multifidus muscle, so most of the extensor momentum of the trunk is generated from the erector spinae muscle, rather than the multifidus muscle. ${ }^{16}$ Dofferhof and Vink ${ }^{17}$ noted that since the erector spinae muscle has a longer lever arm than the multifidus muscle the contraction of the erector spinae muscle in lateral bending motion of the trunk is more effective, and it also plays more important roles for lateral bending during walking. They also pointed out that the activation of the erector spinae muscle helps distribution of the force on the pelvis, facilitating a consistent distribution of the center of mass during walking. McGill ${ }^{18}$ also reported that the erector spinae muscle has appreciable potential to generate torque in all flexion-extension, lateral bending, and axial twisting motions. The erector spinae muscle has the anatomical characteristic of being able to generate more force compared to the multifidus muscle. Therefore, it provides control of the gross trunk movement and general trunk stability.

A sudden and unexpected loading on the lumbar spine can be a potential cause for low back pain, and if lumbar muscles fail to properly react to such a sudden loading, the bending momentum and trunk movement can be greater, which may increase the risk of tissue damages. So, several studies have emphasizing the importance of the erector spinae muscle under these circumstances. ${ }^{19-22}$ When people lift things, the erector spinae muscle plays the key role to generate the torque. ${ }^{19}$ When there is a sudden and unexpected loading on torso, the body's preparation for minimizing the destabilizing postural disturbance and the mechanical loading of the musculoskeletal system always involves the pretensioning of the erector spinae muscle. ${ }^{20}$ The ability of reestablishment of the posture and balance after a sudden and unexpected change in the posture depends on the individuals' ability to rapidly and independently modulate erector spinae muscle. ${ }^{21}$ There are reflex pathways between the erector spinae and lower leg, and the reflexes in erector spinae muscle initiated by the activation of sensory receptors in the foot that provide important information for posture and balance play a role in stabilizing the trunk for maintaining posture and balance. $^{22}$

When acute low back pain occurs in a healthy adult with no past history of low back pain, as in the present study, the CSA of erector spinae muscle at the lower margin of L5 vertebrae in CBP group was observed to be significantly smaller than that of IBP group $(p<0.05)$. While there may be many possible reasons for acute low back pain, most of the cases are thought to be because of sudden and unexpected posture change or loading on the lumbar spine. In these cases, the lumbar erector spinae muscle plays an important role in maintaining the posture and balance by stabilizing the lumbar spine. Therefore, it seems that, for those whose CSA of erector spinae muscle is small, the risk of the developing into chronic low back pain could be higher.

In consideration of the gender ratio difference between the two groups in this study, not only the CSA of erector spinae muscle but also the proportion of the area to the lumbar muscles was measured for comparison. As was the case with the direct comparison of the CSA, the proportion of erector spinae muscle to lumbar muscles at the lower margin of L5 vertebrae was significantly smaller in the CBP group $(\mathrm{p}<0.05)$. This was a comparison of the proportion of the CSA of erector spinae muscle to the total sum of the CSA of lumbar muscles, which showed a statistically significant difference between the two groups without regard to the gender ratio, meaning that there is a association between the development of the acute back pain into a chronic one and the erector sipnae muscle. Also, since the IBP group had a smaller ratio of male patients (12 males and 13 females) compared to the CBP group (10 males and three females), and considering that males typically have larger muscles that females, it can be stated that the possibility of the mean CSA of the erector spinae muscle in the CBP group can be measured relatively larger than that of the IBP group compared to when the ratio between the two genders were equal. This implies that the statistically significantly smaller CSA of erector spinae muscle at the lower margin of L5 vertebrae in CBP group than that of IBP group is a sufficiently meaningful result.

In the present study, the CSA of the multifidus muscle measured at the lower margin of L5 vertebrae did not differ significantly between the CBP and IBP groups. According to Hides et al. ${ }^{10}$ and Wallwork et al., ${ }^{11}$ the CSA 
of the multifidus muscle does not differ significantly between males and females at the L5 vertebral level. So, the results in this study could be regarded as significant, in spite of the gender ratio difference between the two groups. Also, as with the case with the erector spinae muscle, the proportion of multifidus muscle to lumbar muscles was calculated and compared between the two groups. Like the comparison of the CSA, the proportion of the multifidus muscle at the lower margin of L5 vertebrae did not reveal any statistically significant difference between the two groups (Table 3). This is the result of comparison of the proportion of the CSA of the multifidus muscle to the total CSA of lumbar muscles, having sufficient meaning without regard to the gender ratio difference. But, this result is somewhat different from those of previous studies, which focused on the important role of multifiuds muscle in the stabilization of the lumbar spine. There are several explanations for this. For the patients with chronic low back pain, there are two mechanisms that cause the atrophy of the multifidus muscle: disuse and denervation. If multifidus muscle atrophy is caused by disuse, the atrophy should be generalized. But, previous studies have established that multifidus muscle atrophy in the unilateral low back pain is localized rather than generalized. This negates the possibility of disuse atrophy as the basic mechanism. ${ }^{10,11,23,24}$ Kader et al. ${ }^{8}$ concluded that lumbar dorsal ramus syndrome causes multifidus muscle atrophy of patients with low back pain, which triggers a selfsustained vicious cycle that promotes atrophy. Barker et al. ${ }^{24}$ noted that the multifidus muscle atrophy occurs where nerve root compression or irritation occurs, with the degree of atrophy being positively dependent on the duration and severity of the disease. Also, in a study that examined the histochemical changes of the multifidus muscle in the patients of lumbar herniated intervertebral discs, the atrophy of muscle fiber was only observed where there was nerve root impairment. ${ }^{25}$ These findings suggest that the multifidus muscle atrophy in patients with chronic low back pain can be associated with lumbar lesions, such as lumbar herniated intervertebral discs, which could either pressurize or irritate the nerve roots. So, the present study excluded all the patients who had any lumbar lesions on MRI in order to eliminate the influence of the lesions. Thus, the present results are somewhat different from those of previous studies reporting multifidus muscle atrophy in patients with chronic low back pain. Also, while previous studies compared the CSA of lumbar muscles from patients with chronic low back pain with those of the healthy asymptomatic subjects, or those between the affected side and unaffected side in patients with unilateral back pain, this study followed-up the patients who had an lumbar MRI at the earlier stage of low back pain and divided them into two groups by their duration of low back pain and compared the CSA of the lumbar muscles between the two groups, to investigate the association between the chronicity of low back pain and the CSA of lumbar muscles. So, the implications drawn of this study could be regarded as being different from those of previous studies.

In this study, the CSA of erector spinae muscle and the proportion of the area to lumbar muscles at the lower margin of L5 vertebrae in the CBP group were significantly smaller than that of the IBP group. At the lower margin of L3 vertebrae, however, the two groups did not show any statistically significant difference. These findings were because the majority of lumbar spinal movements and weight bearings occur at the lower lumbar level.

The two groups in this study showed no significant difference in the CSA of the psoas muscle at the lower margin of the L3 and L5 vertebrae. The CBP group, however, had a significantly higher proportion of psoas muscle to the lumbar muscles at the lower margin of the L5 vertebrae. This finding seems to result from the relatively lower proportion of erector spinae muscle in the CBP group, because the proportions of the multifidus muscle were almost the same in the two groups (Table 3).

There were some limitations in this study. At first, as was the case with the previous studies, ${ }^{11,13,24}$ the small number of the subjects could be a limitation. There were quite a number of patients who received a lumbar MRI in our Department of Rehabilitation for the 4-year period of the study. However, we excluded all of those with existing lumbar lesions on MRI to eliminate the influence of the lesions. As a result, a large number of patients with low back pain were excluded because of their lumbar lesions, and the study sample size became small. The second limitation is the difference in gender ratio between the two groups. To control this difference, we compared not only the CSA of the erector spinae muscle but also the 
proportion of the area to the lumbar muscles between the two groups. Also, since it is known that there is no gender difference in terms of the CSA of the multifidus muscle at the L5 vertebral level, which is known to be the location of the frequent occurrence of multifidus muscle atrophy and the majority of lumbar spinal movements and weight bearings, ${ }^{10,11,24}$ we did not expect the gender difference would affect the result of this study seriously. But, as it has been shown that the CSA of the multifidus muscle is larger in males than females at the L3 vertebral level, ${ }^{10,11}$ further studies with larger sample size will be necessary to compare male and female subjects.

\section{CONCLUSION}

The CSA of erector spinae muscle and the proportion of it to the lumbar muscles at lower lumbar level can be considered to be prognostic factors concerning chronicity of low back pain. So, we expect that evaluation of CSA of erector spinae muscle and the proportion of the area to the lumbar muscles using MRI will be valuable helpful for further evaluation and planning the treatment of acute low back pain.

\section{REFERENCES}

1. Waddell G. 1987 Volvo award in clinical sciences. A new clinical model for the treatment of low-back pain. Spine 1987; 12: 632-644

2. Cypress BK. Characteristics of physician visits for back symptoms: a national perspective. Am J Public Health 1983; 73: 389-395

3. Andersson GB. Epidemiological features of chronic low-back pain. Lancet 1999; 354: 581-585

4. Croft PR, Macfarlane GJ, Papageorgiou AC, Thomas E, Silman AJ. Outcome of low back pain in general practice: a prospective study. BMJ 1998; 316: 1356 1359

5. O'Sullivan PB, Phyty GD, Twomey LT, Allison GT. Evaluation of specific stabilizing exercise in the treatment of chronic low back pain with radiologic diagnosis of spondylolysis or spondylolisthesis. Spine 1997; 22: 2959-2967

6. Hides JA, Jull GA, Richardson CA. Long-term effects of specific stabilizing exercises for first-episode low back pain. Spine 2001; 26: E243-248
7. Franca FR, Burke TN, Hanada ES, Marques AP. Segmental stabilization and muscular strengthening in chronic low back pain: a comparative study. Clinics 2010; 65: 1013-1017

8. Kader DF, Wardlaw D, Smith FW. Correlation between the MRI changes in the lumbar multifidus muscles and leg pain. Clin Radiol 2000; 55: 145-149

9. Danneels LA, Vanderstraeten GG, Cambier DC, Witvrouw EE, De Cuyper HJ. CT imaging of trunk muscles in chronic low back pain patients and healthy control subjects. Eur Spine J 2000; 9: 266-272

10. Hides J, Gilmore C, Stanton W, Bohlscheid E. Multifidus size and symmetry among chronic LBP and healthy asymptomatic subjects. Man Ther 2008; 13: 43-49

11. Wallwork TL, Stanton WR, Freke M, Hides JA. The effect of chronic low back pain on size and contraction of the lumbar multifidus muscle. Man Ther 2009; 14: 496-500

12. Parkkola R, Rytokoski U, Kormano M. Magnetic resonance imaging of the discs and trunk muscles in patients with chronic low back pain and healthy control subjects. Spine 1993; 18: 830-836

13. Dangaria TR, Naesh O. Changes in cross-sectional area of psoas major muscle in unilateral sciatica caused by disc herniation. Spine 1998; 23: 928-931

14. Bergmark A. Stability of the lumbar spine. A study in mechanical engineering. Acta Orthop Scand Suppl 1989; 230: 1-54

15. Macintosh JE, Bogduk N. 1987 Volvo award in basic science. The morphology of the lumbar erector spinae. Spine 1987; 12: 658-668

16. Bogduk N, Macintosh JE, Pearcy MJ. A universal model of the lumbar back muscles in the upright position. Spine 1992; 17: 897-913

17. Dofferhof AS, Vink P. The stabilising function of the $\mathrm{mm}$. iliocostales and the $\mathrm{mm}$. multifidi during walking. J Anat 1985; 140: 329-336

18. McGill SM. Kinetic potential of the lumbar trunk musculature about three orthogonal orthopaedic axes in extreme postures. Spine 1991; 16: 809-815

19. McGill SM, Norman RW. Partitioning of the L4L5 dynamic moment into disc, ligamentous, and muscular components during lifting. Spine 1986; 11: 666-678

20. Lavender SA, Marras WS, Miller RA. The development 
of response strategies in preparation for sudden loading to the torso. Spine 1993; 18: 2097-2105

21. Boudreau S, Farina D, Kongstad L, Buus D, Redder J, Sverrisdottir E, Falla D. The relative timing of trunk muscle activation is retained in response to unanticipated postural-perturbations during acute low back pain. Exp Brain Res 2011; 210: 259-267

22. Clair JM, Okuma Y, Misiaszek JE, Collins DF. Reflex pathways connect receptors in the human lower leg to the erector spinae muscles of the lower back. Exp Brain Res 2009; 196: 217-227

23. Hides JA, Stokes MJ, Saide M, Jull GA, Cooper DH.
Evidence of lumbar multifidus muscle wasting ipsilateral to symptoms in patients with acute/subacute low back pain. Spine 1994; 19: 165-172

24. Barker KL, Shamley DR, Jackson D. Changes in the cross-sectional area of multifidus and psoas in patients with unilateral back pain: the relationship to pain and disability. Spine 2004; 29: 515-519

25. Yoshihara K, Shirai Y, Nakayama Y, Uesaka S. Histochemical changes in the multifidus muscle in patients with lumbar intervertebral disc herniation. Spine 2001; 26: 622-626 